CASEIRO, R.F.; MARCOS FILHO, J. Métodos para a secagem de sementes de cebola submetidas ao condicionamento fisiológico. Horticultura Brasileira, Brasília, v.23, n.4, p.887-892, out-dez 2005.

\title{
Métodos para a secagem de sementes de cebola submetidas ao condicio- namento fisiológico
}

\author{
Roseli Fátima Caseiro²; Julio Marcos Filho' \\ ${ }^{1}$ USP/ESALQ, Depto. Produção Vegetal, C. Postal 9, 13418-900 Piracicaba-SP; Bolsista CNPq; ${ }^{2}$ Pós-graduanda da USP/ESALQ; \\ E-mail: rosecaseiro@hotmail.com
}

\section{RESUMO}

Há controvérsias quanto à possível reversão de efeitos benéficos do condicionamento fisiológico quando as sementes são desidratadas imediatamente após esse tratamento. A presente pesquisa foi conduzida para avaliar a eficiência de diferentes procedimentos de secagem de sementes de cebola após o condicionamento. Amostras de quatro lotes da cultivar Petroline foram hidrocondicionadas entre duas folhas de papel toalha umedecidas com água a $15^{\circ} \mathrm{C}$, por 48 horas, com posterior secagem rápida em estufa com circulação de ar, a $35-40^{\circ} \mathrm{C}$, por aproximadamente 24 horas; secagem lenta a $20^{\circ} \mathrm{C}$ e umidade relativa de $75 \%$, durante $72 \mathrm{~h}$; incubação das sementes em soluções de polietilenoglicol 8000 (340 g/L de água) a $8^{\circ} \mathrm{C}$, durante $24 ; 72$ ou 120 horas; incubação das sementes em banho-Maria a $40^{\circ} \mathrm{C}$, durante $1 ; 3$ e $5 \mathrm{~h}$; incubação das sementes a $35^{\circ} \mathrm{C}$ por 24 e $48 \mathrm{~h}$. Após a secagem lenta e os procedimentos de incubação, as sementes foram secadas rapidamente, até atingirem teor de água em torno de 6,0 a $8,0 \%$. O potencial fisiológico das sementes foi avaliado por meio dos testes de germinação, índice de velocidade de germinação, envelhecimento acelerado e condutividade elétrica. Adotou-se delineamento experimental inteiramente casualizado, com doze tratamentos e quatro repetições para cada teste. Verificou-se que a incubação em PEG 8000 reduziu drasticamente a germinação e o vigor das sementes condicionadas. Não ocorreram variações acentuadas entre os resultados dos processos de secagem rápida, secagem lenta, incubação em banho-Maria e incubação a $35^{\circ} \mathrm{C}$. No entanto, o processo de secagem rápida foi considerado como o mais adequado após o condicionamento das sementes de cebola, pois além de rápido (o período gasto foi de aproximadamente $24 \mathrm{~h}$ ), é o procedimento de execução mais simples e objetivo.

Palavras-chave: Allium cepa, priming, incubação, germinação, vigor.

\begin{abstract}
Evaluation of methods for drying primed onion seeds

This work was carried out to evaluate drying procedures of primed onion seeds. Samples of four seed lots (cultivar Petroline) were primed between two moistened sheets of germination paper towel, at $15^{\circ} \mathrm{C}$, during $48 \mathrm{~h}$, and then submitted to the following drying processes: fast drying (in oven with air circulation, under 35$40^{\circ} \mathrm{C}$, during approximately $24 \mathrm{~h}$ ); slow drying (seeds kept in a chamber under $20^{\circ} \mathrm{C}$ and $50-60 \%$ relative humidity during $72 \mathrm{~h}$ ); incubation in PEG 8000 solution $\left(340 \mathrm{~g} / \mathrm{L}\right.$ water), under $8^{\circ} \mathrm{C}$, during 24; 72 and $120 \mathrm{~h}$; incubation in a water bath under $40^{\circ} \mathrm{C}$, during $1 ; 3$ and $5 \mathrm{~h}$; and incubation in a chamber under $35^{\circ} \mathrm{C}$, during 24 and 48 h. After the slow drying and the incubation processes, seeds were submitted to fast drying to complete seed desiccation to obtain the moisture content around 6-8\% (fresh weight basis). The physiological potential was evaluated through germination, speed of germination, accelerated aging and electrical conductivity tests. The experiment was carried out in a completely randomized design, with 12 treatments and four replicates for each test. Results showed that the incubation in PEG 8000 solution drastically reduced seed germination and vigor of primed seeds. In addition, there were no wide differences among the process of fast drying, slow drying, incubation in water bath and incubation under $35^{\circ} \mathrm{C}$. The fast drying was considered the best procedure to reduce moisture content of primed onion seeds, with the advantage of being faster (the period required was about 24 h) and more practical.
\end{abstract}

Keywords: Allium cepa, priming, incubation, germination, vigor.

(Recebido para publicação em 20 de maio de 2004 e aceito em 3 de julho de 2005

$\mathrm{O}$ condicionamento fisiológico ("priming") das sementes pode beneficiar diretamente o desempenho de lotes de sementes, ao promover redução do período de germinação e emergência das plântulas ou, indiretamente, ao favorecer a tolerância a condições de estresse após a semeadura como, por exemplo, a baixa disponibilidade de água (FINCH-SAVAGE, 1995). Segundo Pill (1995), esse tratamento consiste na hidratação controlada das sementes, ativando os processos metabólicos necessários para a germinação sem no entanto permitir a protrusão da raiz primária. Ape- sar de sua influência sobre a velocidade, a sincronização e a percentagem de germinação, a utilização comercial do condicionamento fisiológico ainda é relativamente baixa (NASCIMENTO, 1998).

Um dos aspectos fundamentais para possibilitar a comercialização das sementes condicionadas é a redução cuidadosa do teor de água após o tratamento até atingir nível adequado para o armazenamento. Dependendo do procedimento utilizado, pode haver reversão dos benefícios alcançados durante o tratamento. Estudos realizados por Heydecker et al. (1975) e Furutani et al. (1986) mostraram que se- mentes de cebola poderiam ser tratadas e posteriormente secadas até atingir o teor de água inicial, sem reversão dos efeitos benéficos do tratamento. Por outro lado, Haigh et al. (1986) verificaram, para a mesma espécie, que após a secagem ao ar por $24 \mathrm{~h}$ em ambiente de laboratório a $15^{\circ} \mathrm{C}$, houve redução da percentagem de emergência das plântulas em campo; no entanto, para tomate e cenoura, a secagem e o armazenamento das suas sementes por 28 dias não provocaram efeitos prejudiciais.

Essa controvérsia persiste quando são consideradas informações obtidas com outras espécies. Assim, para toma- 
te (ARGERICH et al., 1989) e com alhoporró (ROWSE, 1996), a secagem das sementes após o condicionamento promoveu reversão dos efeitos vantajosos obtidos durante o tratamento. Em tomate verificou-se, inclusive, a perda completa da viabilidade das suas sementes. Da mesma forma, a secagem de sementes de alface, realizada a $21^{\circ} \mathrm{C}$ e $45 \%$ de umidade relativa do ar, não influenciou a velocidade e a percentagem de germinação (GUEDES; CANTLIFFE, 1980); porém, quando a secagem foi conduzida em estufa, a $32^{\circ} \mathrm{C}$, houve reversão dos efeitos proporcionados pelo tratamento. Por outro lado, Eira e Marcos Filho (1990), trabalhando com alface, verificaram que tanto a secagem das sementes em ambiente de laboratório, durante 48 h, como a conduzida em estufa, a $32^{\circ} \mathrm{C}$, por $12 \mathrm{~h}$, não foram prejudiciais para os dois lotes de sementes avaliados.

Desta maneira, verifica-se que os efeitos da secagem pós-condicionamento das sementes dependem do procedimento adotado para a secagem, da espécie considerada e do potencial fisiológico dos lotes utilizados. Visando solucionar essa questão, trabalhos mais recentes têm incluído alternativas para tornar as sementes condicionadas mais resistentes à dessecação. Por exemplo, Bruggink e Van der Toorn (1995) constataram que a tolerância à dessecação de sementes germinadas de pepino pôde ser induzida pela incubação das mesmas em soluções de polietilenoglicol. Procedimentos semelhantes foram utilizados por Bruggink et al. (1999), com redução do período de germinação e manutenção da longevidade similar à das sementes não condicionadas. Neste caso, alguns métodos foram utilizados após o condicionamento fisiológico, incluindo a incubação das sementes em polietilenoglicol, a secagem lenta, a secagem rápida e o choque térmico. A similaridade entre o método para induzir a tolerância das sementes à dessecação durante a germinação e aquele utilizado para prevenir a redução da longevidade de sementes condicionadas indica o envolvimento dos mesmos mecanismos fisiológicos e que a tolerância à dessecação e a longevidade das sementes são realmente características relacionadas.
A disponibilidade de um método prático de secagem sem provocar reversão significativa dos benefícios obtidos durante o condicionamento fisiológico deve apresentar contribuição relevante para a evolução do conhecimento científico e abrir novos caminhos para a pesquisa; além disso, poderia acelerar o aprimoramento da tecnologia adotada por empresas produtoras de sementes e, conseqüentemente, permitir o armazenamento seguro e o aumento da oferta de sementes condicionadas aos produtores de hortaliças. Diante do exposto, o presente trabalho teve por objetivo verificar a eficiência de diferentes procedimentos para a secagem de sementes de cebola condicionadas fisiologicamente.

\section{MATERIAL E MÉTODOS}

O presente trabalho foi realizado no Laboratório de Análise de Sementes da ESALQ. As sementes foram hidrocondicionadas de acordo com procedimento adotado por Caseiro et al. (2004). Foram constituídos doze tratamentos (testemunha e onze procedimentos para a secagem), arranjados em delineamento experimental inteiramente casualizado, com quatro repetições. Amostras de 5,0 g de quatro lotes de sementes de cebola, cv. Petroline, com potencial fisiológico diferente, foram embebidas entre duas folhas de papel toalha, durante 48 horas a $15^{\circ} \mathrm{C}$. Após o condicionamento fisiológico efetuou-se a secagem direta das sementes, mediante os procedimentos: a) secagem rápida em estufa com circulação de ar a $35-40^{\circ} \mathrm{C}$, por aproximadamente $24 \mathrm{~h}$; b) secagem lenta, em câmara a $20^{\circ} \mathrm{C}$ e umidade relativa de $75 \%$, durante $72 \mathrm{~h}$; c) incubação pré-secagem das sementes, em solução de polietilenoglicol 8000 (340 g/L de água) a $8^{\circ} \mathrm{C}$, durante $24 ; 72$ ou $120 \mathrm{~h}$; d) incubação das sementes em banho-Maria a $40^{\circ} \mathrm{C}$ (choque térmico), lacrando-se as sementes em envelopes de alumínio durante uma, três e cinco h; e) secagem rápida por curto período, até que houvesse decréscimo de aproximadamente 6 a 8 pontos percentuais no teor de água, seguida por acondicionamento em recipientes plásticos e incubação a $35^{\circ} \mathrm{C}$ por 24 e 48 h. Após a secagem lenta e os três procedimentos de incubação, as sementes foram secadas rapidamente, da maneira já descrita, até atingirem teor de água em torno de 6,0 a $8,0 \%$.

O potencial fisiológico das sementes submetidas aos diferentes tratamentos foi avaliado com a utilização dos seguintes testes:

\section{Grau de umidade}

Determinado antes e após os tratamentos, de acordo com as Regras para Análise de Sementes (BRASIL, 1992), utilizando-se o método da estufa a $105^{\circ} \mathrm{C} \pm 3^{\circ} \mathrm{C}$, por $24 \mathrm{~h}$, com duas repetições de $1,0 \mathrm{~g}$ de sementes para cada lote.

\section{Germinação}

Testes de germinação foram conduzidos com quatro repetições de 50 sementes para cada lote, distribuídas sobre papel chupão (Germibox) e colocadas para germinar a $20^{\circ} \mathrm{C}$. O substrato foi umedecido com volume de água equivalente a 2,5 vezes a sua massa. As contagens foram realizadas diariamente até o $12^{\circ}$ dia após a semeadura, computando-se o número de plântulas normais, sendo os resultados expressos em percentagem média por tratamento e lote.

\section{Velocidade de germinação}

Teste realizado conjuntamente com o de germinação. As avaliações foram realizadas diariamente, à mesma hora, a partir do dia em que foram identificadas as primeiras plântulas normais, que foram computadas e retiradas do substrato. Ao final, com os dados diários do número de plântulas normais foi calculado o índice de velocidade de germinação (IVG), de acordo com a fórmula proposta por Maguire (1962). Os dados foram expressos em índices médios de velocidade de germinação para cada lote.

\section{Envelhecimento acelerado}

Foram utilizadas caixas plásticas $(11,0 \times 11,0 \times 3,5 \mathrm{~cm})$ adaptadas, funcionando como compartimento individual ou mini-câmara. Em cada uma, foram colocados $40 \mathrm{ml}$ de solução saturada de $\mathrm{NaCl}$, conforme Jianhua e McDonald (1996). As sementes foram distribuídas uniformemente sobre a tela de alumínio colocada no interior de cada caixa, formando uma camada única. As caixas 
Tabela 1. Porcentagem de germinação (G), índice de velocidade de germinação (IVG), envelhecimento acelerado (EA), condutividade elétrica (CE) e grau de umidade (GU) de sementes de cebola, cultivar Petroline, lote 1, submetidas à diferentes processos de secagem após o condicionamento fisiológico. Piracicaba, ESALQ, 2002.

\begin{tabular}{|c|c|c|c|c|c|}
\hline Tratamentos & G (\%) & IVG & EA (\%) & $\mathrm{CE} \mu \mathrm{mho} / \mathrm{cm}^{-1} \mathbf{g}^{-1}$ & GU (\%) \\
\hline Testemunha & $86 \mathrm{a}$ & $7,60 \mathrm{cde}$ & 61 cdef & $202,78 \mathrm{e}$ & 5,6 \\
\hline Condicionamento sem secagem & $85 a$ & $12,07 \mathrm{a}$ & 65 bcde & $74,75 a b$ & 44,9 \\
\hline Secagem rápida & $86 a$ & $8,90 \mathrm{bcd}$ & 79 a & $92,05 \mathrm{bcd}$ & 7,9 \\
\hline Secagem lenta & $83 a$ & $9,23 \mathrm{bc}$ & 70 abcd & $114,19 \mathrm{~d}$ & 7,2 \\
\hline Banho maria 1 hora & $88 a$ & $9,52 \mathrm{bc}$ & $76 a b$ & $104,32 \mathrm{bcd}$ & 7,4 \\
\hline Banho maria 3 horas & $90 \mathrm{a}$ & $10,33 \mathrm{~b}$ & $80 \mathrm{a}$ & $107,54 \mathrm{~cd}$ & 7,5 \\
\hline Banho maria 5 horas & $89 a$ & $9,71 \mathrm{bc}$ & $79 a b$ & $102,60 \mathrm{bcd}$ & 7,8 \\
\hline Incubação PEG 24 horas & $81 \mathrm{a}$ & $7,26 \mathrm{efg}$ & 58 def & $81,08 \mathrm{abcd}$ & 7,2 \\
\hline Incubação PEG 72 horas & $79 a$ & $6,69 \mathrm{fg}$ & $49 \mathrm{f}$ & $65,66 \mathrm{a}$ & 6,9 \\
\hline Incubação PEG 120 horas & $80 a$ & $6,16 \mathrm{~g}$ & 50 ef & $69,61 \mathrm{a}$ & 7,2 \\
\hline Incubação $35^{\circ} \mathrm{C} 24$ horas & $86 a$ & 7,67 def & $74 a b c$ & $104,82 \mathrm{bcd}$ & 6,6 \\
\hline Incubação $35^{\circ} \mathrm{C} 48$ horas & $87 \mathrm{a}$ & $8,69 \mathrm{cde}$ & $78 \mathrm{ab}$ & $107,33 \mathrm{~cd}$ & 6,9 \\
\hline C.V (\%) & 5,73 & 6,82 & 6,51 & 12,63 & \\
\hline
\end{tabular}

plásticas foram tampadas e mantidas em uma câmara de envelhecimento (tipo B.O.D.) regulada a $41^{\circ} \mathrm{C} \pm 0,3^{\circ} \mathrm{C}$, onde permaneceram por $72 \mathrm{~h}$. Após esse período, foram colocadas para germinar a $20^{\circ} \mathrm{C}$ e avaliadas aos seis dias após a semeadura. Os resultados foram expressos em percentagem de plântulas normais para cada lote. Avaliações do grau de umidade, antes e após o período de envelhecimento, permitiram verificar a precisão dos resultados.

\section{Condutividade elétrica}

O método utilizado foi o de condutividade de massa, de acordo com Vieira (1994). Foram avaliadas quatro repetições de 50 sementes para cada lote. Cada repetição foi pesada com precisão de duas casas decimais e, a seguir, colocada em um copo plástico com capacidade para $200 \mathrm{ml}$, contendo $50 \mathrm{ml}$ de água

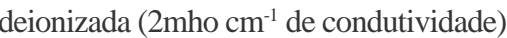
e mantida em germinador a $20^{\circ} \mathrm{C}$, durante $24 \mathrm{~h}$. Após esse período efetuaram-se as leituras da condutividade elétrica da solução de embebição, em condutivímetro Digimed-CD-20 e os dados foram expressos em mmho $\mathrm{cm}^{-1} \mathrm{~g}^{-1}$ de sementes.

Os dados obtidos nesses testes foram submetidos à analise de variância e as médias comparadas pelo teste de Tukey $(\mathrm{p} £ 0,05)$.

\section{RESULTADOS E DISCUSSÃO}

O teste de germinação (Tabelas 1 a 4) não foi suficientemente sensível para detectar variações no potencial fisiológico das sementes submetidas aos diferentes procedimentos para a secagem, com ou sem incubação prévia; as diferenças existentes foram identificadas por todos os testes de vigor conduzidos nesta pesquisa, normalmente mais rigorosos que o de germinação.

As sementes apenas condicionadas apresentaram índice de velocidade de germinação (IVG) sempre significativamente superior ao das sementes submetidas ao hidrocondicionamento e secagem, para todos os lotes avaliados (Tabelas 1 a 4). Essa ocorrência pode ser atribuída à necessidade de um período adicional para que as sementes com menor grau de umidade atingissem níveis semelhantes aos das mais úmidas, durante a etapa inicial do processo de germinação. Verificou-se que as sementes não submetidas à secagem apresentaram grau de umidade em torno de $45 \%$, enquanto, após a secagem, esses valores atingiram aproximadamente $7 \%$. Sabe-se que a velocidade de germinação está diretamente relacionada ao grau de umidade da semente (ROSSETTO et al., 1997). No entanto, mesmo após a secagem, os valores do IVG foram superiores aos obtidos para a testemunha, indicando que a secagem não promoveu a reversão dos efeitos benéficos do condicionamento, quando avaliados pela velocidade de germinação.

A incubação em polietilenoglicol durante 24; 72 e $120 \mathrm{~h}$ e a conduzida a $35^{\circ} \mathrm{C}$, durante 24 e $48 \mathrm{~h}$, para todos os lotes avaliados, foram os tratamentos que conduziram aos menores IVG, quando comparados aos demais métodos (Tabelas 1 a 4). Em contrapartida, os IVG mais elevados foram observados após a incubação em banho-Maria, durante 3 e 5 h. Entretanto, neste caso, não foi detectada diferença significativa em relação ao processo de secagem rápida, que foi considerado o método mais rápido e prático.

Considerando o comportamento das sementes condicionadas e não secadas, no teste de envelhecimento acelerado (Tabelas 1 a 4), verificou-se que, em presença de solução saturada de $\mathrm{NaCl}$, as que inicialmente apresentavam grau de umidade em torno de $45,1 \%$ (média dos quatro lotes), foram cedendo água para o ambiente durante o teste e, no final, o seu teor de água permaneceu ao redor de $12,7 \%$ (média dos quatro lotes). Desta maneira, as sementes não permaneceram com grau de umidade elevado durante todo o teste, o que contribuiu para amenizar os efeitos do envelhecimento acelerado. De acordo com Marcos Filho (1999), as sementes mais úmidas geralmente são mais sensíveis às condições desse teste. Bruggink et al. (1999) observaram que, após o teste de deterioração controlada, houve redução da porcentagem de germinação das não secadas após o condicionamento; vale destacar que os princípios dos testes de envelhecimento acelerado e de deterioração controlada são equivalentes.

De maneira geral, foi constatado que as sementes condicionadas e secadas 
Tabela 2. Porcentagem de germinação (G), índice de velocidade de germinação (IVG), envelhecimento acelerado (EA), condutividade elétrica (CE) e grau de umidade (GU) de sementes de cebola, cultivar Petroline, lote 2, submetidas à diferentes processos de secagem após o condicionamento fisiológico. Piracicaba, ESALQ, 2002.

\begin{tabular}{|c|c|c|c|c|c|}
\hline Tratamentos & G (\%) & IVG & EA (\%) & $\mathrm{CE} \mu \mathrm{mho} / \mathrm{cm}^{-1} \mathbf{g}^{-1}$ & GU (\%) \\
\hline Testemunha & $85 \mathrm{a}$ & $8,02 \mathrm{de}$ & $73 a b$ & $179,18 \mathrm{~d}$ & 6,6 \\
\hline Condicionamento sem secagem & $85 a$ & $13,75 \mathrm{a}$ & 76 a & 66,74 a & 44,2 \\
\hline Secagem rápida & $87 a$ & $10,29 \mathrm{bc}$ & 81 a & $88,77 \mathrm{c}$ & 7,7 \\
\hline Secagem lenta & $87 a$ & $10,28 \mathrm{bc}$ & $58 \mathrm{bc}$ & $90,21 \mathrm{c}$ & 7,3 \\
\hline Banho maria 1 hora & $80 a$ & $9,10 \mathrm{~cd}$ & 78 a & $95,58 \mathrm{c}$ & 7,1 \\
\hline Banho maria 3 horas & $81 a$ & $10,73 \mathrm{~b}$ & $73 a b$ & $95,14 \mathrm{c}$ & 7,6 \\
\hline Banho maria 5 horas & $84 \mathrm{a}$ & $10,29 \mathrm{bc}$ & 79 a & $90,60 \mathrm{c}$ & 7,7 \\
\hline Incubação PEG 24 horas & $80 a$ & $8,11 \mathrm{de}$ & $52 \mathrm{c}$ & $73,11 a b$ & 6,6 \\
\hline Incubação PEG 72 horas & $82 \mathrm{a}$ & 7,49 de & $58 \mathrm{bc}$ & 60,45 a & 6,9 \\
\hline Incubação PEG 120 horas & 76 a & $7,05 \mathrm{e}$ & $48 \mathrm{c}$ & 64,65 a & 6,8 \\
\hline Incubação $35^{\circ} \mathrm{C} 24$ horas & $80 a$ & $7,86 \mathrm{de}$ & $74 a b$ & $93,58 \mathrm{c}$ & 6,1 \\
\hline Incubação $35^{\circ} \mathrm{C} 48$ horas & $82 \mathrm{a}$ & $8,37 \mathrm{de}$ & 76 a & $87,65 \mathrm{bc}$ & 6,9 \\
\hline C.V (\%) & 5,97 & 7,03 & 7,45 & 6,97 & \\
\hline
\end{tabular}

Tabela 3.. Porcentagem de germinação (G), índice de velocidade de germinação (IVG), envelhecimento acelerado (EA), condutividade elétrica (CE) e grau de umidade (GU) de sementes de cebola, cultivar Petroline, lote 3, submetidas à diferentes processos de secagem após o condicionamento fisiológico. Piracicaba, ESALQ, 2002.

\begin{tabular}{|c|c|c|c|c|c|}
\hline Tratamentos & G (\%) & IVG & EA (\%) & CE $\mu \mathrm{mho} / \mathrm{cm}^{-1} \mathbf{g}^{-1}$ & GU (\%) \\
\hline Testemunha & $89 a$ & $9,02 \mathrm{fg}$ & 77 bcde & $185,26 \mathrm{e}$ & 5,3 \\
\hline Condicionamento sem secagem & $92 \mathrm{a}$ & $14,24 \mathrm{a}$ & $85 \mathrm{abcd}$ & 61,58 a & 45,8 \\
\hline Secagem rápida & $89 a$ & $10,71 \mathrm{bcd}$ & $87 \mathrm{abc}$ & $81,60 \mathrm{bc}$ & 7,6 \\
\hline Secagem lenta & 90 a & $10,54 \mathrm{~cd}$ & 80 abcde & $83,28 \mathrm{bc}$ & 7,3 \\
\hline Banho maria 1 hora & $92 \mathrm{a}$ & $10,37 \mathrm{cde}$ & 83 abcd & $90,46 \mathrm{~cd}$ & 7,1 \\
\hline Banho maria 3 horas & 93 a & $11,93 \mathrm{~b}$ & $87 a b$ & $92,42 \mathrm{~cd}$ & 7,6 \\
\hline Banho maria 5 horas & 93 a & $11,52 \mathrm{bc}$ & 89 a & $100,60 d$ & 7,9 \\
\hline Incubação PEG 24 horas & $88 \mathrm{a}$ & $8,90 \mathrm{fg}$ & 75 cde & $73,92 a b$ & 6,8 \\
\hline Incubação PEG 72 horas & $91 \mathrm{a}$ & $8,93 \mathrm{fg}$ & $67 \mathrm{e}$ & 64,55 a & 6,9 \\
\hline Incubação PEG 120 horas & $83 a$ & $7,76 \mathrm{~g}$ & $73 \mathrm{de}$ & $69,38 a b$ & 7,0 \\
\hline Incubação $35^{\circ} \mathrm{C} 24$ horas & 94 a & 9,86 def & 84 abcd & $95,35 \mathrm{~cd}$ & 6,6 \\
\hline Incubação $35^{\circ} \mathrm{C} 48$ horas & $90 \mathrm{a}$ & 9,15 ed & $85 \mathrm{abcd}$ & 84,46 bc & 6,9 \\
\hline C.V (\%) & 5,78 & 5,33 & 5,75 & 7,18 & \\
\hline
\end{tabular}

foram mais resistentes às condições de estresse de alta temperatura e alta umidade relativa do ar, as quais foram impostas pelo teste de envelhecimento acelerado. As sementes submetidas aos procedimentos de secagem rápida e à incubação em banho-Maria por 1; 3 e 5 h ou em estufa a $35^{\circ} \mathrm{C}$ por $48 \mathrm{~h}$ (Tabela 1 ) e as sementes submetidas ao banho-maria por $5 \mathrm{~h}$ (Tabela 3), apresentaram germinação significativamente superiores à testemunha.

Na presente pesquisa, os procedimentos utilizados para a secagem das sementes de cebola, foram baseados nos por Bruggink et al. (1999), que obtiveram resultados semelhantes com a secagem lenta, banho-Maria (ou choque térmico) e incubação das sementes a $35^{\circ} \mathrm{C}$; todos esses procedimentos beneficiaram o potencial fisiológico das sementes.

Ao contrário do verificado no presente trabalho, Bruggink et al. (1999) verificaram que a incubação das sementes de Impatiens walleriana Hook em PEG 8000 foi eficiente para prevenir a redução do potencial de armazenamento. Esse mesmo procedimento, utilizado por Bruggink e Van der Toorn (1995), havia induzido a tolerância à dessecação de sementes germinadas de pepino. Por outro lado, Bruggink et al. (1999) mencionaram que as sementes de amor-perfeito e de pimentão submetidas à secagem rápida, foram mais sensíveis ao teste de deterioração controlada em relação às submetidas à secagem lenta e à incubação a $35^{\circ} \mathrm{C}$, respectivamente. Conseqüentemente, verifica-se que a eficiência dos métodos para induzir tolerância à dessecação e/ou melhor desempenho das sementes durante o armazenamento das sementes também é influenciada pela espécie utilizada.

Quando a eficiência dos procedimentos de secagem foi avaliada pelo teste de condutividade elétrica, todos os métodos provocaram redução significativa na exudação de solutos quando comparados à testemunha. Vários autores têm sugerido que o condicionamento fisiológico pode exercer efeito benéfi- 
Tabela 4. Porcentagem de germinação (G), índice de velocidade de germinação (IVG), envelhecimento acelerado (EA), condutividade elétrica (CE) e grau de umidade (GU) de sementes de cebola, cultivar Petroline, lote 4, submetidas à diferentes processos de secagem após o condicionamento fisiológico. Piracicaba, ESALQ, 2002.

\begin{tabular}{|c|c|c|c|c|c|}
\hline Tratamentos & G (\%) & IVG & EA (\%) & $\mathrm{CE} \mu \mathrm{mho} / \mathrm{cm}^{-1} \mathbf{g}^{-1}$ & GU (\%) \\
\hline Testemunha & $93 a$ & 9,62 ef & $82 \mathrm{abc}$ & $156,90 \mathrm{f}$ & 6,2 \\
\hline Condicionamento sem secagem & 94 a & 16,08 a & $87 a b$ & $63,53 a b$ & 45,4 \\
\hline Secagem rápida & 94 a & $11,66 \mathrm{bc}$ & $88 \mathrm{ab}$ & $76,55 \mathrm{~cd}$ & 7,6 \\
\hline Secagem lenta & $92 a$ & $11,14 \mathrm{~cd}$ & $88 a b$ & $84,55 \mathrm{de}$ & 7,2 \\
\hline Banho maria 1 hora & $92 \mathrm{a}$ & $10,89 \mathrm{cde}$ & 91 a & 89,68 e & 7,1 \\
\hline Banho maria 3 horas & $94 a$ & $12,86 \mathrm{~b}$ & $78 \mathrm{bc}$ & 91,81 e & 7,4 \\
\hline Banho maria 5 horas & $92 \mathrm{a}$ & $12,02 \mathrm{bc}$ & $86 a b$ & 91,94 e & 7,5 \\
\hline Incubação PEG 24 horas & $93 a$ & 9,74 ef & $76 \mathrm{bc}$ & $71,80 \mathrm{bc}$ & 6,4 \\
\hline Incubação PEG 72 horas & $86 a$ & $8,70 \mathrm{f}$ & $70 \mathrm{c}$ & 62,07 a & 6,6 \\
\hline Incubação PEG 120 horas & $88 a$ & $8,80 \mathrm{f}$ & $76 \mathrm{bc}$ & 61,56 a & 6,5 \\
\hline Incubação $35^{\circ} \mathrm{C} 24$ horas & $92 \mathrm{a}$ & $10,24 \mathrm{de}$ & $87 a b$ & 87,94 e & 6,1 \\
\hline Incubação $35^{\circ} \mathrm{C} 48$ horas & $93 a$ & 9,93 def & $89 a b$ & $78,13 \mathrm{~cd}$ & 6,4 \\
\hline C.V (\%) & 6,24 & 4,77 & 6,21 & 4,49 & \\
\hline
\end{tabular}

co sobre a integridade das membranas celulares (WOODSTOK; TAO, 1981; ARMSTRONG; MCDONALD, 1992). Porém, em outros trabalhos, foi ressaltado que a redução na lixiviação de solutos após o condicionamento fisiológico pode ocorrer principalmente em função da perda de eletrólitos durante esse tratamento e talvez não esteja relacionada à reorganização das membranas (DEARMAN et al., 1986; CHOJNOWSKI et al., 1997). No entanto, conforme destacaram Woodstock e Tao (1981), durante o processo de condicionamento fisiológico, a hidratação ocorre de maneira mais lenta, aumentando a disponibilidade de tempo para o reparo ou reorganização gradual das membranas.

Os valores da condutividade elétrica obtidos após a execução de todos os métodos de secagem (Tabelas 1 a 4), exceto incubação em PEG 8000, foram superiores aos observados para as sementes não secadas. Neste caso, a secagem provavelmente provocou desorganização do sistema de membranas, permitindo maior lixiviação de eletrólitos. Porém, observa-se que após todos os procedimentos de secagem houve redução significativa na lixiviação de solutos, quando comparados à testemunha, indicando a ocorrência de reparo no sistema de membranas durante o condicionamento fisiológico e que, mesmo após a secagem, pelo menos parte desse reparo e/ou reorganização foi mantida. No caso da incubação das sementes em PEG 8000, ressalta-se que,

Tabela 5. Grau de umidade (\%) após o período de incubação pré- secagem, após o condicionamento fisiológico de quatro lotes de sementes de cebola, cultivar Petroline. Piracicaba ESALQ, 2002

\begin{tabular}{lccccc}
\hline \multirow{2}{*}{ Tratamentos } & \multicolumn{5}{c}{ Lotes } \\
\cline { 2 - 6 } & $\mathbf{1}$ & $\mathbf{2}$ & $\mathbf{3}$ & $\mathbf{4}$ & Média \\
\hline Secagem lenta & 10,1 & 10,5 & 10,0 & 10,0 & 10,2 \\
Banho maria 1 hora & 39,3 & 38,9 & 38,3 & 38,9 & 38,9 \\
Banho maria 3 horas & 40,3 & 39,3 & 41,1 & 39,7 & 40,1 \\
Banho maria 5 horas & 34,7 & 35,0 & 39,1 & 35,7 & 36,1 \\
Incubação PEG 24 horas & 47,6 & 47,3 & 48,2 & 48,0 & 47,8 \\
Incubação PEG 72 horas & 48,1 & 49,5 & 48,7 & 49,1 & 48,9 \\
Incubação PEG 120 horas & 48,4 & 48,9 & 49,3 & 48,8 & 48,9 \\
Incubação 35 $\mathrm{C}$ 24 horas & 39,2 & 38,1 & 40,2 & 39,7 & 39,3 \\
Incubação 35 $\mathrm{C}$ 48 horas & 33,6 & 32,4 & 34,6 & 29,2 & 32,5 \\
\hline
\end{tabular}

ao contrário dos demais métodos, as sementes ficaram imersas em solução $\mathrm{e}$, por isso, novamente pode ter ocorrido perda de eletrólitos no momento da incubação, diminuindo ainda mais a quantidade de lixiviados durante o teste de condutividade elétrica.

No teste de condutividade elétrica para todos os lotes avaliados,com exceção do tratamento de incubação em PEG 8000 (Tabelas 1 a 4), a secagem rápida foi o método que provocou as menores perdas de solutos durante o teste; destaca-se ainda que as sementes submetidas ao banho-maria por cinco horas (Tabela 3) e as submetidas a banho-Maria por $1 ; 3$ e 5 h e à incubação a $35^{\circ} \mathrm{C}$, por $24 \mathrm{~h}$ (Tabela 4), apresentaram maiores índices de lixiviação em relação às secadas rapidamente. De maneira geral, a incubação das sementes em PEG 8000, durante $24 ; 72$ e $120 \mathrm{~h}$, foi o procedimento menos favorável à manifestação do potencial fisiológico das sementes, não promovendo a tolerância à dessecação.

Com exceção da incubação em PEG 8000 (Tabela 5), os demais procedimentos provocaram decréscimo no grau de umidade das sementes condicionadas, que apresentavam em torno de $45,1 \%$ de água (média dos quatro lotes) antes do início da incubação. No entanto, esses valores permaneceram elevados quando foi efetuada a incubação em solução de PEG 8000. Isto justifica o desempenho inferior das sementes expostas a essa modalidade de incubação antes da secagem definitiva, pois as sementes mais úmidas apresentam atividade metabólica mais intensa, predispondo-as à deterioração. 
Desta maneira, as sementes de cebola foram sensíveis à desidratação após o condicionamento fisiológico, apenas quando foram mantidas com grau de umidade acima de $47,3 \%$, por período superior a $24 \mathrm{~h}$, antes da secagem. No método de incubação em solução de PEG 8000, as sementes que já haviam iniciado as atividades metabólicas mais intensas durante o condicionamento fisiológico, foram ainda incubadas por períodos de 24; 72 e $120 \mathrm{~h}$, sem que houvesse condições para a continuidade do processo de germinação ou o início da secagem, permanecendo por mais tempo com teores elevados de água. De acordo com Vertucci e Farrant (1995), as células naturalmente tolerantes à dessecação, quando mantidas com graus de umidade associados à ocorrência de atividades catabólicas, podem sofrer danos mais severos se permanecerem nessas condições durante período prolongado, quando comparadas às secadas rapidamente. Assim, segundo aquelas autoras, a capacidade de tolerância das sementes à dessecação também depende da maneira como a secagem é realizada.

Os dados obtidos no presente trabalho demonstraram que é possível efetuar a secagem após o condicionamento fisiológico de sementes de cebola, sem provocar reversão significativa dos efeitos favoráveis ao potencial fisiológico. Como não foram verificadas variações acentuadas entre os processos de secagem rápida, secagem lenta, incubação em banho-Maria e incubação a $35^{\circ} \mathrm{C}$, o uso da secagem rápida é mais conveniente para a redução do grau de umidade das sementes condicionadas de cebola pois, além de rápido, esse procedimento é mais prático que os demais métodos que envolvem incubação.

\section{LITERATURA CITADA}

ARMSTRONG, H.; McDONALD, M.B. Effects of osmoconditioning on water uptake and electrical conductivity in soybean seeds. Seed Science \& Technology, v.20, p.391-400, 1992.

BRASIL. Ministério da Agricultura e Reforma Agrária. Regras para análise de sementes. Brasília: SNDA/DNDV/CLAV, 1992. 365 p. BROCKLEHURST, P.A.; DEARMAN, J. Interactions between seed priming treatments and nine seed lots of carrot, celery and onion. I. Laboratory germination. Annals of Applied Biology, v.102, p.577-584, 1983.

BRUGGINK, G.T.; OOMS, J.J.J.; Van der TOORN, P. Induction of longevity in primed seeds. Seed Science Research, v.9, p.49-53, 1999. BRUGGINK, T.; van der TOORN, P. Induction of desiccation tolerance in germinated seeds. Seed Science Research, v.5, p.1-4, 1995.

CASEIRO, R.F.; BENNETT, M.A.; MARCOS FILHO, J. Comparison of three priming techniques for onion seed lots differing in initial seed quality. Seed Science and Technology, v.32, n.2, p.365-375, 2004.

CHOJNOWSKI, M.; CORBINEAU, F.; CÔME, D. Physiological and biochemical changes induced in sunflower seeds by osmopriming and subsequent drying, storage and aging. Seed Science Research, v.7, p.323-331, 1997.

DEARMAN, J.; BROCKLEHURST, P.A.; DREW, R.L. Effects of osmotic priming and ageing on onion seed germination. Annals of Applied Biology, v.108, p.639-648, 1986.

EIRA, M.T.S.; MARCOS-FILHO, J. Condicionamento osmótico de sementes de alface. I. Efeitos sobre a germinação. Revista Brasileira de Sementes, Brasília, v.12, n.1, p.9-27, 1990.

FINCH-SAVAGE, W.E. Influence of seed quality on crop establishment, growth, and yield. In: BASRA, A.S. (Ed.). Seed quality: basic mechanisms and agricultural implications. Binghamton, NY: The Haworth Press, 1995. p.361-384.

FURUTANI, S.C.; ZANDSTRA, B.H.; PRICE, H.C. The effects of osmotic solute composition and duration and temperature of priming on onion seed germination. Seed Science \& Technology, v.14, p.545-551, 1986.

GUEDES, A.C.; CANTLIFFE, D.J. Germination of lettuce seeds at high temperature after seed priming. Journal of the American Society for Horticultural Science, v.105, n.6, p.777-781, 1980.
HAIGH, A.M.; BARLOW, E.W.R.; MILTHORPE, F.L. Field emergence of tomato, carrot, and onion seeds primed in an aerated salt solution. Journal of the American Society for Horticultural Science, v.111, n.5, p.660-665, 1986. HEYDECKER, W.; HIGGINS, J.; TURNER, Y.J. Invigoration of seeds. Seed Science \& Technology, v.3, p.881-888, 1975.

JIANHUA, Z.; McDONALD, M.B. The saturated salt accelerated aging test for small-seeded crops. Seed Science \& Technology, v.25, p.123-131, 1996.

MAGUIRE, J.D. Speed of germination-aid in selection and evaluation for seedling emergence and vigor. Crop Science, v.2, n.1, p.176-177, 1962. MARCOS-FILHO, J. Teste de envelhecimento acelerado. In: KRZYZANOWSKI, F.C.; VIEIRA, R.D.; FRANÇA-NETO, J.B. (Eds.). Vigor de sementes: conceitos e testes. Londrina: Abrates, 1999. p.1-24.

NASCIMENTO, W.M. Condicionamento osmótico de sementes de hortaliças: potencialidades e implicações. Horticultura Brasileira, Brasília, v.16, n.2, p.106-109, 1998.

PILL, W.A. Low water potential and presowing germination treatments to improve seed quality In: BASRA, A.S. (Ed.). Seed quality: basic mechanisms and agricultural implications. Binghamton, NY: The Haworth Press, 1995, cap.10, p.319-359.

ROSSETO, C.A.V.; NOVEMBRE, A.D.L.C.; MARCOS FILHO, J.; SILVA, W.R.; NAKAGAWA, J. Comportamento das sementes de soja durante a fase inicial do processo de germinação. Scientia Agrícola, Piracicaba, v.54, n.1/ 2, p.106-115, 1997.

ROWSE, H.R. Drum priming - A non-osmotic method of priming seeds. Seed Science \& Technology, v.24, p.281-294, 1996.

VERTUCCI, C.W.; FARRANT. J.M. Acquisition and loss of desiccation tolerance. In: KIGEL, J. \& GALILI, G. (Eds.). Seed development and germination. New York: Marcel Dekker, 1995. p.237-271.

VIEIRA, R.D. Teste de condutividade elétrica. In: VIEIRA, R.D.; CARVALHO, N.M. (Eds.). Testes de vigor em sementes. Jaboticabal: Funep, 1994. p.103-132

WOODSTOCK, L.W.; TAO, K.J. Prevention of imbibitional injury in low vigor soybean embryonic axes by osmotic control of water uptake. Physiologia Plantarum, v.51, p.133-139, 1981. 